\title{
A Study on Beam Extraction Characteristics of RF and DC Filament Ion Source for High Current Ion Implanters
}

Received 11 March, 2021; revised 4 May, 2021; accepted 21 May, 2021

\author{
Ju-Hong Cha, Sang-Woo Kim, and Ho-Jun Lee* \\ Department of Electrical Engineering, Pusan National University, Busan 46241, Republic of Korea \\ *Corresponding author E-mail: hedo@pusan.ac.kr
}

\begin{abstract}
In high current implantation, improving the ion extractability is a decisive factor contributing to the equipment efficiency and process cost reduction. A direct current filament ion source and inductively coupled plasma ion source were prepared to observe the degree of plasma extraction according to the ion source characteristics. The plasma parameters used to optimize beam extraction for the filament and RF discharges were $600 \mathrm{~W}$, Ar $2 \times 10^{-5}$ Torr, and Ø200 $\times 250 \mathrm{~mm}$ discharge space. In the thermionic ion source, the ion density, electron temperature, and Bohm current density were $5 \times 10^{9} 1 / \mathrm{cm}^{3}, \geq 6 \mathrm{eV}$, and $\approx 3.5 \mathrm{~A} / \mathrm{m}^{2}$, respectively. In the RF ion source, the ion density, electron temperature, and Bohm current density were approximately $5 \times 10^{10} 1 / \mathrm{cm}^{3}, \approx 3 \sim 4 \mathrm{eV}$, and $\approx 25 \mathrm{~A} / \mathrm{m}^{2}$, respectively. Optimization and analysis of ion extraction according to the plasma and accelerator parameters were calculated using IBSimu. The beam emittance, which is the most important parameter of the ion beam quality, was measured as the beam envelope onto the phase space by an ellipse. A change in the plasma meniscus shape was observed according to the plasma parameters in the ion source, and the emittance of the extracted ion beam was calculated according to the electrode gap distance and aperture structure.
\end{abstract}

Keywords: High current ion implant, Inductively coupled plasma, Hot cathode filament discharge, Ion extraction

\section{Introduction}

In high current low energy ion-implantation equipment used for S/D contact or poly-Si gate doping, the long-term operational stability of the ion beam source and the ion beam current extracted from the ion source are the main factors affecting the process productivity. In high current ion implant equipment used in ion implantation for semiconductor processing, thermionic discharged ion sources are commonly used for plasma generation. Thermionic discharged ion sources are based on electron emission from a hot cathode to produce the desired plasma density through ionization of the background gas. The process is suitable for use in low-pressure $\left(1 \times 10^{-5}\right.$ Torr $)$ processes owing to the electron-emitting discharge mechanism. The use of a filament is essential for cathode heating, but corrosion of the filament due to heating leads to a decrease in their lifetime and performance. As the development of a high performance and long life ion source is required, studies on RF inductively coupled plasma (ICP) ion sources, which can be driven at low pressures, are being conducted actively [1] The ICP plasma source is driven by the induced electric field generated by the time-varying magnetic field, and relatively high ionization efficiency is achieved because of the low energy losses in the sheath and wall at the power coupling mechanism. In order to improve the productivity compared to the previously used ion source, ion sources with plasma properties higher than electron density $5 \times 10^{10} 1 / \mathrm{cm}^{3}$ and bohm current density $25 \mathrm{~A} / \mathrm{m}^{2}$ are required. For the increase of ion beam efficiency, structural optimization in the form of emittance smaller than ' 10 ' is also required. The ion source lifetime using the RF ion sources $(\approx 1000 \mathrm{hr}$ ) can be improved two fold compared to that of

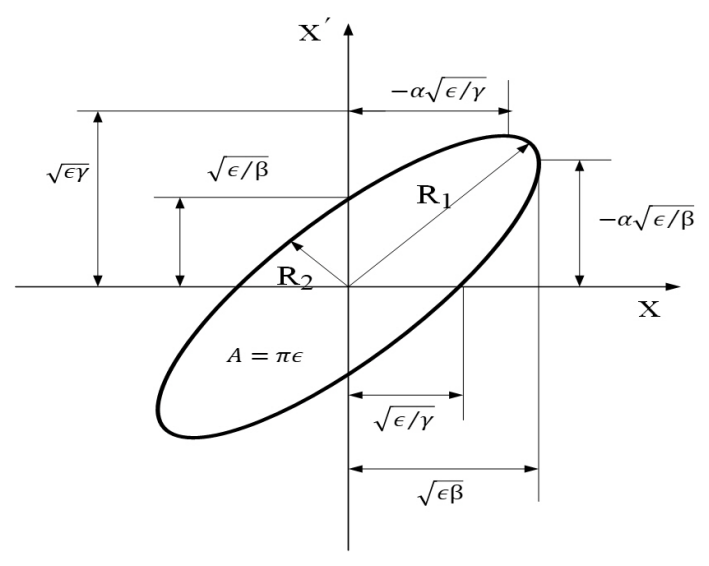

Figure 1. Geometry of the emittance ellipse with the dimensions.

filament-type ion sources $(\approx 500 \mathrm{hr})$. To increase the ion beam current extracted from the ion source, two methods that increase the electrical power applied during discharge in the ion source and optimize the beam extraction in the accelerator are needed [2]. On the other hand, the increase in applied power is limited because of the tradeoff relationship with contamination inside the chamber. Therefore, it is essential to optimize ion beam extraction to prevent chamber contamination problems and increase the extractability of ions [3]. In this study, the plasma parameters used to optimize beam extraction 


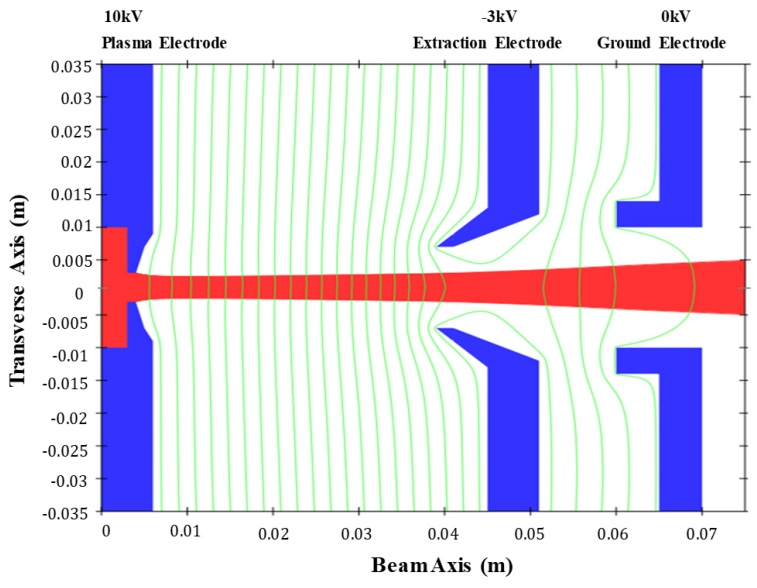

Figure 2. Geometry and ion beam trajectory in IBSimu.

for filament and RF discharges were based on $600 \mathrm{~W}$ (applied power), pressure of $2 \times 10^{-5}$ Torr (Ar gas), and discharge space of Ø200 $\times$ $250 \mathrm{~mm}$. Extraction was based on the three-electrode system consisting of a plasma electrode, extraction electrode, and ground electrode, which are commonly used in high current ion implantation equipment [4]. The optimization and analysis of ion extraction according to the plasma and accelerator parameters were calculated using IBSimu (Ion Beam Simulation). IBSimu is an optical computer simulation used for the optimization and design of ion-source extraction systems. This code can also be used to analyze the negative and positive ion extraction, but the most significant advantage is the relatively accurate calculation of the ion extraction boundary of the plasma source and space charge dominated ion beam transport. Analytical scaling and numerical calculations of ion extraction from plasma are difficult because of the nonlinearity by the plasma into Poisson's equation. As an alternative, a simple method for approximating based on Kovaleski's hypothesis that the sheath edge from an unmagnetized plasma coincides with a critical electric field magnitude [5]. The plasma sheath surface over the aperture is called the meniscus, whose shape and surface area are dependent on the plasma density and temperature. The beam optics depends on the plasma density and electron temperature of the plasma source for the given electrode geometry. The extraction surface area and topology can be determined using $|E|=E_{p}=T_{e} / \lambda_{D}$ ( $T_{e}$ : electron temperature, $\lambda_{D}$ : Debye length, $E_{p}$ : critical electric field magnitude) with a single Laplace solution of the extraction system [5]. The initial ion current and ion optical conditions can be calculated, assuming that the Bohm current density describes the ion extraction current, and the ions are extracted along a vector perpendicular to the surface. The results of this calculation system were reported to be well matched with the PIC simulations results in high current ion sources. In this study, calculations of the two dimensional cylindrical symmetry geometry were conducted using the plasma parameters obtained from the experimental results as the plasma conditions (plasma potential, ion current, and electron temperature) in the simulation. In the thermionic ion source using a hot cathode filament discharge, ion density, electron temperature, and Bohm current density were $5 \times 10^{9}$ $1 / \mathrm{cm}^{3}, \geq 6 \mathrm{eV}$, and $\approx 3.5 \mathrm{~A} / \mathrm{m}^{2}$, respectively. On the other hand, with the RF ion source, which has relatively high ionization efficiency, the ion density, electron temperature, and Bohm current density were approximately $5 \times 10^{10} 1 / \mathrm{cm}^{3}, \approx 3 \sim 4 \mathrm{eV}$, and $\approx 25 \mathrm{~A} / \mathrm{m}^{2}$, respectively. The plasma meniscus changes according to the ion current density, sheath depth, and $E_{p}$ value with ion-source aperture geometry depending on the type of ion source. This study analyzed the differences in the ion optics caused by the change in plasma meniscus along the plasma parameters and source characteristics between the filament type and RF ion sources. (a)

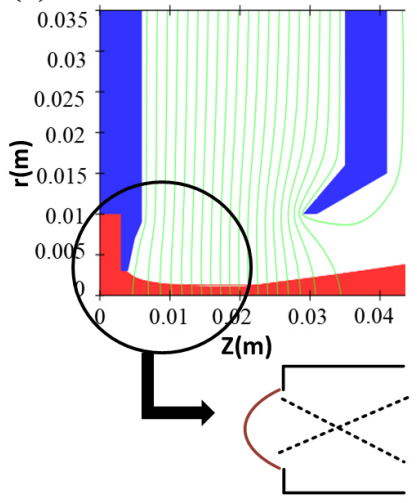

(b)

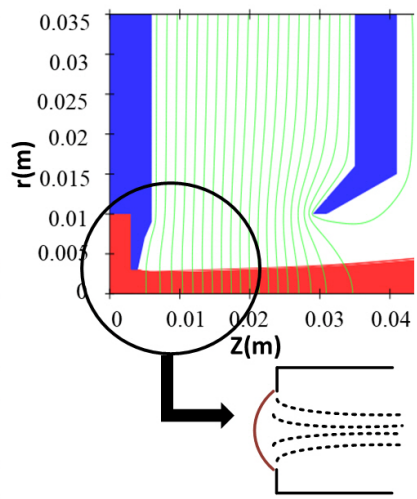

Figure 3. Two simulations of $13 \mathrm{keV} \mathrm{Ar}^{+}$extraction with varying plasma meniscus. (a) Beam extraction from the filament discharge source (concave meniscus). (b) Beam extraction from the RF ion source (cross-over meniscus).

\section{Extraction simulation and discussion}

The beam emittance is one of the important parameters among the optical characteristics of the beam. With practical accelerators, the important measurement in the ion beam quality is the volume of the beam envelope. In the case where forces acting on the particles are linear, the phase space of the particle is considered by the normalized momenta $\left(x^{\prime}, y^{\prime}, z^{\prime}\right)$ with respect to the synchronous particle. As the longitudinal direction of the beam is of less interest, transverse distributions $\left(x, x^{\prime}\right)$ were used instead of the full phase space distribution for simplicity. When modeling the beam distributions, the projections of the distribution onto the phase plane were represented ideally by an ellipse.

$$
\begin{aligned}
& \gamma x^{2}+2 \alpha x x^{\prime}+\beta x^{\prime} 2=\epsilon \\
& A=\pi \epsilon=\pi R_{1} R_{2} \\
& \beta \gamma^{2}-\alpha^{2}=1
\end{aligned}
$$

In Eqs. (1)-(3), $\epsilon$ is the two dimensional transverse emittance, and $\alpha, \beta$, and $\gamma$ are the Twiss parameters, which are used to describe the emittance ellipse. $A$ is the area of the ellipse, and $R_{1}$ and $R_{2}$ are the major and minor radii of the ellipse, respectively.

Figure 2 presents the ion beam trajectory composed of three different electrodes on a two-dimensional cylindrical symmetry geometry. The first electrode terminates the plasma surface, and the plasma electrode plays an important role in the optical characteristics of the beam. The shape of the emission aperture and plasma meniscus is determined here. The second extraction electrode has a negative potential compared to the plasma potential that extracts and accelerates the ion beams. The gap distance between the first and second electrodes, electric field direction formed by the inclined angle, and applied DC voltage of the extraction electrode help determine the ion beam optics. In this simulation, the simulation parameters were set to $\mathrm{Ar}^{+}$ion at a $13 \mathrm{kV}$ extraction voltage, gap distance of $33 \mathrm{~mm}$, particle trajectory number of 50000, mesh size of $0.05 \mathrm{~mm}$, and iteration number of 15 .

Figure 3 presents the result of a ion-beam extraction simulation for the DC filament discharge ion source and RF ion source. In Fig. 3(a), where the ion beam is extracted from the filament discharge source, a concave meniscus appears, and the beam proceeds in the converging direction. In Fig. 3(b), the ion beam extracted from the RF ion source proceeds as parallel, and a slightly concave meniscus shape is achieved [6]. Because electric potential difference of RF aperture wall is much thinner than the filament-type ion source, a higher quality beam, which proceeds in the direction normal to the equipotential plane, can be achieved. In the filament-discharge ion source with a 


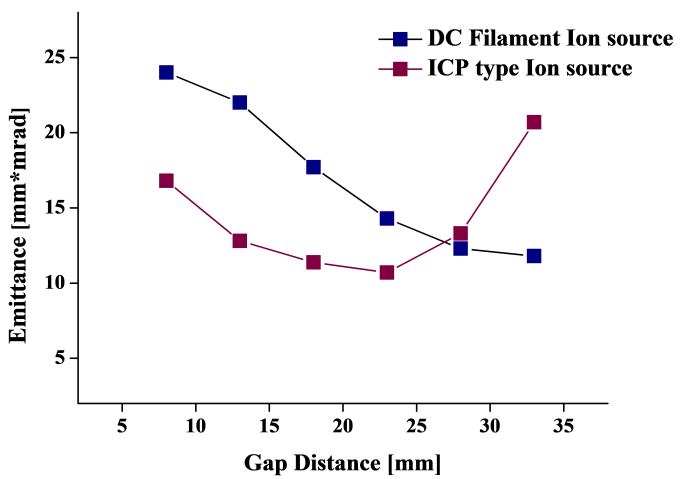

Figure 4. Variation of the emittance versus gap distance.

relatively low electron density, the depth of electric potential difference is thick, so the plasma meniscus shape formed on the aperture surface is critical to the ion beam optics.

Figure 4 shows the variation of the emittance versus the gap distance between the plasma electrode and the extraction electrode without a change in the other conditions. The beam emittance, $\epsilon$, is the product of $R_{1}$ and $R_{2}$ (major and minor radii of the ellipse), and describes the two dimensional transverse emittance. The ion beam propagation in the traveling direction and the degree of divergence in the radial direction are expressed as the emittance, $\epsilon$ [7]. The beam, which has a smaller $\epsilon$ value, has better beam extractability. In the beam line, the extraction electrode serves to extract the beam from the ion source. Sometimes, the secondary electrode affects the shape change in the equipotential line to diverge the beam, as shown in Fig. 2. Because of the slightly concave meniscus shape at the source aperture, the high quality ion beam can be extracted from the RF ion source. The change in the ion beam properties according to the structure and the radius curvature of the plasma electrode is relatively small, and the beam extractability according to the position and structure of the secondary electrode is more important [8]. As shown in Fig. 4, in the RF source, as the gap distance is increased, the emission decreases and then increases. When the electrode gap distance between the first and second electrode is $23 \mathrm{~mm}$, the emittance is a minimum, and the beam extraction performance is optimized. In the DC filament ion source, the emittance is a minimum, where the gap distance is $35 \mathrm{~mm}$, and the beam optics are influenced relatively little by the secondary electrode shape [9]. The beam property extracted from the DC filament ion source is affected dominantly by the plasma meniscus on the aperture surface. As shown in Fig. 5, the geometry of the aperture surface determines the beam emittance. The investigation of the beam emittance based on the angle of inclination affecting the field formation at the aperture surface revealed a high quality beam at 45 degrees.

\section{Conclusions}

In the trajectory composed of three different electrodes on a two dimensional cylindrical symmetry geometry, the simulation of the beam transport of a $13 \mathrm{keV} \mathrm{Ar}{ }^{+}$beam was conducted using IBSimu. The plasma sheath surface over the aperture called the meniscus is dependent on the plasma density and temperature. The ion beam extracted from the filament-discharge source results in the formation of a concave meniscus, and the beam proceeds in the converging direction. The ion beam extracted from the RF ion source has a slightly concave meniscus shape. A higher quality beam can be achieved because the sheath depth at the RF aperture wall is much thinner than the filament ion source, and the meniscus shape is slightly concave at the source aperture. When using RF ion sources, the change in the ion

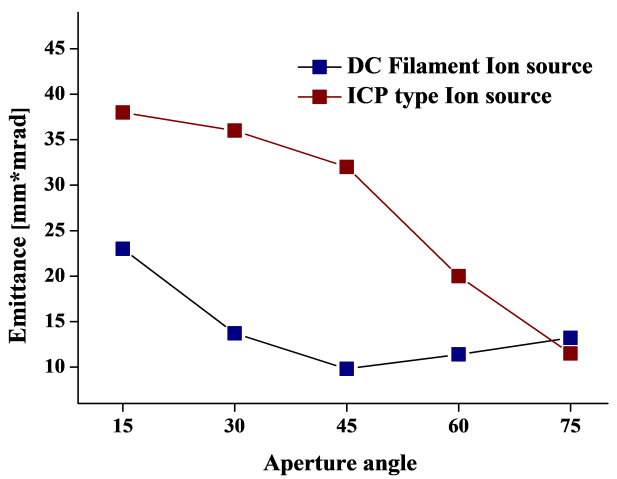

Figure 5. Variation of the emittance versus aperture angle.

beam properties according to the structure of the plasma electrode is relatively small, and the beam extractability described by the position and structure of the secondary electrode is more important. On the other hand, the plasma meniscus on the aperture surface has a major effect on the beam property extracted from the DC filament ion source. Therefore, optimization of the aperture geometry is most important for generating a high quality beam discharge.

\section{Acknowledgements}

This research was supported by Korea Institute for Advancement of Technology (KIAT) grant funded by the Korea Government (MOTIE) (P0012451, The Competency Development Program for Industry Specialist).

\section{References}

[1] M. A. Lieberman and A. J. Lichtenberg, Principles of Plasma Discharges and Materials Processing, 2nd Ed. (John Wiley \& Sons, Inc., 2005).

[2] B. Wolf, Handbook of Ion Sources (CRC Press, 1995).

[3] I. G. Brown, The physics and Technology of Ion Sources, 2nd Ed. (WILEY-VCH Verlag GmbH \& Co. KGaA, Weinheim, 2004).

[4] T. Kalvas, O. Tarvainen, T. Ropponen, O. Steczkiewicz, J. Ärje, and H. Clark, Rev. Sci. Instrum. 81, 02B703 (2010).

[5] S. D. Kovaleski, IEEE Trans. Plasma Sci. 34, 23 (2006).

[6] O. Sutherland, J. Keller, M. Irzyk, and R. Boswell, Rev. Sci. Instrum. 75, 2379 (2004)

[7] M. Khoshhal, M. Habibi, and R. W. Boswell, Laser Part. Beams 36, 477 (2018).

[8] R. Becker and W. B. Herrmannsfeldt, Rev. Sci. Instrum. 63, 2756 (1992).

[9] T. Kalvas, S. K. Hahto, F. Gicquel, M. King, J. H. Vainionpää, J. Reijonen, K. N. Leung, and T. G. Miller, Rev. Sci. Instrum. 77, 03B904 (2006). 